Meta

Journal des traducteurs

Translators' Journal

\title{
Enseignement de la terminologie : une pratique pédagogique
}

\section{Marthe Faribault}

Volume 33, numéro 2, juin 1988

L'enseignement de la traduction au Canada - Teaching Translation

in Canada

URI : https://id.erudit.org/iderudit/002724ar

DOI : https://doi.org/10.7202/002724ar

Aller au sommaire du numéro

Éditeur(s)

Les Presses de l'Université de Montréal

ISSN

0026-0452 (imprimé)

1492-1421 (numérique)

Découvrir la revue

Citer cet article

Faribault, M. (1988). Enseignement de la terminologie : une pratique pédagogique. Meta, 33(2), 299-303. https://doi.org/10.7202/002724ar d'utilisation que vous pouvez consulter en ligne.

https://apropos.erudit.org/fr/usagers/politique-dutilisation/ 


\section{ENSEIGNEMENT DE LA TERMINOLOGIE : UNE PRATIQUE PÉDAGOQIQUE}

MARTHE FARIBAULT

Université de Montréal, Montréal, Québec

\section{LES COURS DE TERMINOLOGIE À L'U. DE M.}

Les étudiants des programmes de baccalauréat en traduction doivent suivre obligatoirement deux cours de terminologie ( 90 heures). Les étudiants inscrits au programme de mineur en traduction (1 ${ }^{\mathrm{er}}$ cycle) ne suivent que le premier cours (45 heures). Les étudiants du baccalauréat en linguistique peuvent suivre, en option, un cours de terminologie ; un cours indépendant des programmes de traduction a été conçu spécialement pour cette clientèle. Au niveau de la maîtrise, un programme de linguistique appliquée, spécialisé en terminologie, est dispensé ; ce programme contient quelques cours communs avec celui de la maîtrise en traduction et plusieurs cours couvrent le domaine de la terminologie sous ses divers aspects. Enfin, la maîtrise en traduction comporte un séminaire de terminologie de 45 heures.

Je ne traiterai ici que des cours de terminologie qui s'adressent aux étudiants inscrits à la maîtrise en traduction.

\section{LA MAÎTRISE EN TRADUCTION : CLIENTĖLES ÉTUDIANTES}

Les étudiants des options francophones et anglophones suivent les mêmes cours de terminologie. L'année de maîtrise proprement dite accueille directement les étudiants ayant obtenu un diplôme de premier cycle en traduction. Les étudiants qui ont fait un baccalauréat dans une autre discipline doivent faire au préalable une année propédeutique, dite de " prémaîtrise ". Le cours d'Introduction à la terminologie est obligatoire en prémaîtrise. L'année de scolarité proprement dite comporte un séminaire obligatoire de terminologie.

À l'entrée en prémaîtrise, la formation en linguistique des étudiants varie dans tous les azimuts, puisque ces étudiants peuvent aussi bien venir d'un programme spécialisé de linguistique que d'un programme de langue étrangère (où ils ont acquis des notions générales de linguistique, sans toutefois avoir nécessairement suivi de cours de linguistique proprement dits) ou d'un programme de sciences pures ou de sciences humaines (où l'enseignement de la linguistique et des langues est absent). À ces étudiants s'ajoutent ceux du mineur en traduction qui ont, à l'entrée au programme, au plus deux années d'études de niveau baccalauréat dans une quelconque matière (généralement linguistique, langues étrangères ou littérature). S'ajoutent encore les étudiants ayant obtenu un baccalauréat en traduction dans une autre université, mais dont le programme ne comporte aucun cours de terminologie.

Dans leur programme de prémaîtrise ou de mineur, tous nos étudiants doivent suivre obligatoirement un cours de Théorie de la traduction; ils peuvent prendre, en option, des cours de Grammaire contrastive et de Sémantique. Tous les autres cours du programme sont des cours de rédaction, de traduction et de langue de spécialité.

À l'entrée du séminaire de maîtrise, les étudiants ont une formation très variable en linguistique. Du point de vue de la terminologie, ils peuvent avoir déjà suivi deux cours (baccalauréat de traduction incluant deux cours de terminologie) ou un seul cours 
(tous les autres). Dans le cadre des cours à option, ils peuvent suivre des séminaires de Traduction automatique et de Théorie de la traduction. Tous les autres séminaires portent sur la traduction.

\section{LES COURS DE TERMINOLOGIE À LA MAÎTRISE EN TRADUCTION}

\subsection{L'objectif des cours}

Il est double. D'abord initier les étudiants au domaine général de la terminologie, puisque certains d'entre eux peuvent trouver des postes de terminologues, plutôt que de traducteurs, à la fin de leurs études. Ensuite, de rendre les traducteurs en herbe aptes à régler les problèmes de terminologie posés par les textes à traduire, en les aidant à devenir plus critiques face aux ouvrages lexicographiques et terminographiques qu'ils utilisent quotidiennement et en les rendant aptes à une recherche originale, pour les cas problèmes. À la maîtrise, le cours d'Introduction à la terminologie (prémaîtrise) et le Séminaire de terminologie (maîtrise) sont donnés en parallèle avec des cours de traduction spécialisée ou de langue de spécialité, auxquels ils servent de complément. Le séminaire sert également de préparation au travail de recherche de maîtrise (travail dirigé ou mémoire), pour les étudiants qui choisiront de faire une étude de terminologie, théorique ou pratique, en vue de l'obtention de leur diplôme. Schématiquement, ces cours de terminologie dispensés dans le cadre d'un programme de traduction doivent donner aux étudiants une solide méthode de travail, des notions de base en terminologie et lexicologie et une certaine connaissance du fonctionnement, de l'utilisation et de l'alimentation des banques de termes.

\subsection{Le contenu}

Le contenu d'un cours de terminologie à objectifs aussi larges et s'adressant à des clientèles aussi diversifiées est difficile à établir. Idéalement, il faudrait tout couvrir, en 90 heures. Ce contenu général, qui devrait faire d'un étudiant un bon terminologue débutant, a déjà été décrit à diverses reprises au cours des dix dernières années par d'autres professeurs de terminologie, tant canadiens qu'européens (Auger, Dubuc, Roberts, Sager, Picht). Je ne le répéterai pas ici ; je vais plutôt exposer de façon concrète la solution pédagogique à laquelle je suis arrivée après quelques années d'enseignement. Je m'attarderai davantage sur le premier cours, d'introduction, que sur le second, qui m'apparaît comme le plus important, puisqu'il est à la base de tout le travail qui se fera ultérieurement et qu'il est peut-être le plus difficile à mettre au point.

\section{DESCRIPTION DU COURS D'INTRODUCTION À LA TERMINOLOGIE}

Étant donné la diversité de la clientèle étudiante, surtout à la prémaîtrise, j’ai organisé le cours en deux blocs : un bloc de thèmes fixes, qui contient la matière systématiquement enseignée tous les ans, et un bloc de thèmes variables, qui sont traités cycliquement et plus ou moins longuement, suivant la formation antérieure des étudiants, leurs intérêts, et suivant les questions de l'heure dans les domaines de la recherche terminologique et des politiques linguistiques. Pour ce dernier bloc de cours, on peut dire que, de façon générale, les exposés organisés selon une trame historique (histoire du français au Québec, histoire des banques de termes, etc.) sont très appréciés des étudiants et amènent des questions intéressantes et des discussions animées.

En début de trimestre, la terminologie est présentée du point de vue de la traduction. Ce point de vue est progressivement déplacé pour en venir vers le milieu du trimestre à traiter de la terminologie comme d'une activité en soi, le point de vue de la traduction passant en arrière-plan. Le cours est organisé suivant deux niveaux : 
1. en classe, des exposés magistraux du professeur suivis d'exercices d'application ;

2. en dehors des heures de cours, un petit travail de recherche thématique de 25 fiches, suivi en tutorat, du début à la fin du trimestre.

L'objectif de ce cours reste assez limité : acquisition de quelques notions de base et d'une bonne méthode de travail. Au cours des dernières années, j’ai expérimenté deux méthodes pédagogiques dans le cadre de ce cours.

La première, que je qualifierai de "bain global " consiste à lancer les étudiants d'emblée dans un travail de terminographie, en leur fournissant dès le début du trimestre un protocole de rédaction de fiches terminologiques (protocole-maison ou directives du Manuel de R. Dubuc, dérivés du protocole de l'ancienne Banque de terminologie de l'Université de Montréal, ou protocoles de la BTQ ou de la BTC), avec un minimum de préparation méthodologique et théorique, comptant sur les questions soulevées en cours de trimestre par les travaux individuels des étudiants et les exercices en classe pour approfondir les notions de base. C'est une méthode de sensibilisation efficace qui a pour avantage de donner aux étudiants, dès le départ, une image précise du travail qu'ils devront soumettre en fin de trimestre. Elle a cependant pour inconvénient majeur de donner une image quelque peu faussée du travail de recherche terminologique, mettant davantage l'accent sur les règles d'écriture des données terminologiques, plutôt que sur les questions de fond.

J'ai abandonné cette méthode depuis quelques années pour adopter la seconde, beaucoup plus satisfaisante à mon point de vue, compte tenu des objectifs du programme. Elle se résume à ceci :

- aucun protocole de rédaction n'est fourni;

- les étudiants travaillent avec une fiche de dépouillement, et non avec une fiche de synthèse destinée au stockage définitif des données;

- la trame du cours repose sur les notions de base;

- les règles d'écriture des données terminologiques sont présentées progressivement, comme découlant des questions de fond exposées. Elles sont également présentées comme une forme de solution à une situation de communication, avec les trois degrés possibles : fichier personnel, fichier manuel commun d'un groupe de travail, fichier informatisé à contraintes strictes.

Cette méthode a l'avantage de remettre la question de l'écriture des données terminologiques à sa juste place (c'est-à-dire à celle d'une simple convention d'écriture, plus ou moins stricte suivant la situation de communication) et de ramener les problèmes de base à l'avant-plan. Elle a encore comme avantage de rendre les étudiants adlaptables à n'importe quelle convention d'écriture qui pourrait leur être imposée par la suite. Quant aux inconvénients, on peut souligner que les étudiants restent avec une vision floue du travail qu'ils ont à exécuter jusqu'à la mi-trimestre, où ils ont le sentiment de "enfin commencer à comprendre "; qu'ils ne se rendent compte qu'en fin de trimestre de l'importance de certaines questions vues au début du trimestre. Mais, plus qu'un inconvénient, ceci m'apparaît comme un indice de cours d'introduction réussi : que les étudiants, en fin de trimestre, aient envie de tout reprendre depuis le début signifie qu'ils ont acquis une bonne vue d'ensemble de la matière couverte. Voici la structure de ce cours d'introduction. 


\subsection{Les cours magistraux}

\subsubsection{Les thèmes fixes \\ DOCUMENTATION}

Exposé :

- Règles d'écriture de la fiche bibliographique

- Ressources documentaires de la ville de Montréal et du campus

- Visite du centre de documentation du Département

- Systèmes de classification par matières des bibliothèques

- Systèmes de classification par domaines des données terminologiques

- Typologie d'une documentation de base en recherche terminologique

- Bibliographie de base de la terminologie

Règles d'écriture de la fiche terminologique :

- Inscription des sources bibliographiques des données terminologiques

Exercice :

- Inscription des identifications de domaine

- Rédaction d'une courte bibliographie suivant une règle donnée

- Courte recherche documentaire au centre de documentation du Département

- Attribution d'identification de domaine à des termes de domaines divers

\section{DÉPOUILLEMENT : LE TERME}

\section{Exposé :}

- Différence entre unité terminologique et unité de discours

- Caractéristiques des vocabulaires spécialisés :

- Limites entre vocabulaire de la langue générale, vocabulaire technique et scientifique général, vocabulaire spécialisé

- Caractéristiques sémantiques : monosémie/polysémie; structures générique/spécifique et partie/tout des lexiques; représentation par des arborescences

- Caractéristiques morphologiques : importance de la dérivation suffixale à base verbale; problème particulier des nominalisations; structure des syntagmes complexes et problème des critères de lexicalisation

\section{Règles d'écriture :}

- Conventions générales d'écriture des vedettes

- Traitement des unités verbales et adjectivales

Exercice :

Inscription des catégories grammaticales

- Ȧ partir d'un texte terminologique mixte :

- repérer tous les termes n'appartenant pas au vocabulaire général

- trier les termes suivant différentes identifications de domaine spécialisé, dont le vocabulaire technique et scientifique général

- discuter au fur et à mesure, suivant la séquence du texte, les cas de termes complexes (unités de lexique ou de discours) et de nominalisations (lexicales ou phrastiques)

- souligner au passage les cas de cooccurrence

\section{DÉPOUILLEMENT - LA JUSTIFICATION \\ Exposé :}

- Définition de dictionnaire de langue générale, de dictionnaire spécialisé et définition en discours 
- Contextes plus ou moins informatifs, par opposition à la définition en discours

- Contextes métalinguistiques : caractéristiques formelles; conventions typographiques

- Questions de choix de justification

Règles d'écriture :

- Longueur de la justification

- Règles de troncation et d'élucidation d'éléments

- Identification des types de justifications

Exercice :

- À partir d'un texte de type pédagogique ou encyclopédique :

- identification des définitions en discours et des contextes métalinguistiques

- écriture de justification sur les fiches, avec identification du type de justification

- Recherche et comparaison de définitions tirées de dictionnaires de langue générale, de lexiques spécialisés et de textes

- Comparaison de justifications de divers types pour un terme donné

MISE EN ÉQUIVALENCE

Exposé :

- Qualité relative des justifications

- Preuve d'équivalence

- Cas d'équivalences imparfaites et de lacunes lexicales

Règles d'écriture :

Exercice :

- Marques d'usage sémantique

- Analyse de fiches bilingues du point de vue du recoupement des justifications

- Confection de fiches à partir de deux textes anglais et français sur un sujet donné ; recherche de justifications complémentaires dans une documentation plus large

\section{TRAITEMENT DES SYNONYMES}

Exposé :

- Variation lexicale internationale, régionale, sociale, locale, individuelle; variation temporelle

- Les contextes synonymiques. Problèmes d'ambiguïté des contextes " ou/or"

\section{Règles d'écriture :}

- Justification des synonymes

- Marques d'usage : codes conventionnels

Rédaction des observations terminologiques

Exercices :

- Sur texte, repérage et analyse de contextes synonymiques. Discussion sur tous les cas d'ambiguité de contexte "ou/or" et sur les génériques anaphoriques

- Lecture de textes en jargon technique

4.1.2 Les thèmes variables

L'OFFICE DE LA LANGUE FRANÇAISE

- Histoire des politiques linguistiques au Québec et mandat de l'OLF

- Développement de la Banque de terminologie du Québec 
- Présentation du matériel de la BTQ : fiche de travail et fiche informatisée

- Séance d'interrogation de la BTQ

\section{LE SECRÉTARIAT D'ÉTAT}

- Histoire de la politique du bilinguisme au gouvernement canadien

- Développement de la Banque de terminologie de l'U. de M. et de la Banque de terminologie du Canada

- Présentation du matériel de la BTC : fiches de travail et fiches informatisées

- Séance d'interrogation de la BTC

\section{LE MILIEU PROFESSIONNEL}

- Exposé d'un professionnel en exercice, terminologue ou professionnel effectuant des travaux de terminologie en contexte de traduction ou de francisation.

\section{LA COOCCURRENCE}

- Caractéristiques

- Techniques de dépouillement et de classification

- Utilité de ce type de dépouillement pour le traducteur

LA NORMALISATION

- Normalisation et contextes de communication

- Organismes de normalisation internationaux, nationaux, locaux

- Etude de documents normatifs

\subsection{Le travail de recherche thématique}

Les étudiants doivent fournir en fin de trimestre une recherche thématique de 25 fiches (ou dossiers), accompagnée d'une bibliographie et d'un court travail de synthèse. On leur suggère de travailler en groupe de deux ou trois, ce qui leur permet de voir un plus grand nombre de cas, sans pour autant augmenter leur quantité de travail. On leur souligne que le travail en équipe, lorsque bien mené, amène une recherche plus efficace, plus approfondie et une certaine forme d'autocorrection.

Les instructions générales pour ce travail sont données en classe. Des mises au point sont effectuées périodiquement ; des périodes de questions sont ouvertes régulièrement, pendant les heures de cours. En dehors de cela, la recherche est dirigée par tutorat, en parallèle avec les cours en classe. La séquence est la suivante :

\section{DÉBUT DE TRIMESTRE}

Les étudiants ont trois semaines pour mettre en place leur sujet de recherche, avec les conseils suivants :

\section{Choix du sujet}

On recommande aux étudiants de s'orienter vers des sujets concrets, qui amènent une terminologie relativement limitée et cohérente. Ainsi, on leur recommande : en terminologie technique :

- terminologie relative à un appareil : différents types et parties composantes

- terminologie relative à une catégorie d'instruments

- terminologie relative à un procédé de fabrication : différentes opérations, appareils nécessaires, différents états de la matière en terminologie scientifique :

- biologie : terminologie relative à un organisme vivant ou à un organe particulier

- sciences de la terre : terminologie relative à un type de relief particulier, à un type d'érosion des sols; océanographie ; météorologie 
On recommande aux étudiants de ne pas s'orienter vers un vocabulaire abstrait (vocabulaires des sciences humaines) ni vers le vocabulaire d'une science de base (vocabulaire des mathématiques, de la physique, de la chimie), à moins qu'ils n'aient déjà eux-mêmes une formation dans l'un de ces domaines. Ceci à cause des problèmes posés par la délimitation des concepts et par les variations de terminologie d'une école de pensée à l'autre. Pour ce premier travail de recherche terminologique, on n'insiste pas tant sur l'originalité du travail que sur sa systématicité : couverture d'un ensemble de termes structuré et recherche de synonymes.

\section{Repérage documentaire}

En tenant compte des directives données au cours dans le bloc DOCUMENTATION, les étudiants doivent constituer et présenter au professeur une bibliographie de base pour leur sujet de recherche. L'accent est mis sur la qualité et la quantité de la documentation en français, étant donné les lacunes habituelles à ce niveau. Si, pour un sujet donné, la documentation française présentée après trois semaines de repérage est insuffisante, l'étudiant doit, suivant le jugement du professeur, soit changer de sujet, soit compléter sa recherche en une semaine.

\section{Établissement de la nomenclature de départ}

Pour éviter que l'étudiant ne se perde dans un sujet trop vaste et pour l'aider à constituer un corps de vocabulaire cohérent, on lui demande de trouver un " texte de départ " sur lequel il effectuera un dépouillement terminologique. On demande à l'étudiant de trouver un texte qui lui fournisse une cinquantaine de termes propres à son domaine d'étude, ce qui lui donne une marge de mancuvre par rapport aux 25 fiches à fournir. Ce dépouillement est critiqué avec chaque étudiant par le professeur pour éliminer les " termes qui ne sont pas des termes " (vocabulaire général, unités de discours), souligner la structuration du vocabulaire repéré, dans le but d'orienter la suite de la recherche et souligner les vocabulaires de domaines connexes, s'il y a lieu.

\section{MI-TRIMESTRE}

Une remise de 10 fiches bilingues complètes, accompagnées d'une bibliographie et du texte de départ, est demandée. Une correction systématique de la forme et du fond est effectuée, en vue d'orienter la suite du travail. Les défauts habituels à cette première remise sont :

- conventions typographiques non respectées

- fonction des différents champs de la fiche non respectée

- identification des types de justification pas comprise

- mauvaise qualité des justifications

- méconnaissance du latin, en vocabulaire médical

- fausses synonymies

- manque de recherche au niveau des synonymes

- difficulté à saisir l'existence et l'importance de la variation linguistique, d'où mauvaise utilisation des marques d'usage

- équivalences fausses

- équivalences justes, mais sans preuve par manque de recoupement entre les justifications

- mise en équivalence de mots ou expressions "qui se ressemblent ", et non des notions, d'où équivalences fausses

- mise en équivalence de termes de catégories grammaticales différentes, d'où équivalences fausses 
- mélange de plusieurs notions sur une même fiche (cas des termes polysémiques)

- observations terminologiques pas faites ; observations qui n'ont pas un propos linguistique ; marques métalinguistiques pas ou mal utilisées dans la rédaction des observations

- copiage de documents lexicographiques bilingues, sans autre forme de recherche

Les défauts identifiés dans ce premier travail sont discutés individuellement avec chaque étudiant. Un certain nombre de travaux corrigés sont montrés en classe sur opascope, pour expliquer les défauts typiques et pour discuter les cas problèmes, avec les solutions possibles.

À la suite de cette première correction, les étudiants sont en général assez familiers avec leur sujet et avec la méthodologie générale pour pouvoir élargir leur stratégie de recherche. On leur permet de s'éloigner de leur texte de départ, pour viser une meilleure systématicité dans leur étude terminologique.

\section{FIN DU TRIMESTRE}

Les étudiants doivent remettre

- les 10 fiches du premier travail corrigées et complétées

- 15 nouvelles fiches

- la bibliographie complète

- une courte rédaction ( 3 pages pour un travail individuel ; 5 pages pour une équipe) qui fasse la synthèse du travail du trimestre, aux niveaux suivants :

- délimitation du sujet de la recherche

- critique de la documentation rassemblée

- caractérisation du vocabulaire étudié : vocabulaire spécifique au domaine et vocabulaire des domaines connexes ; vocabulaire ancien ; nombreux néologismes ; unités complexes nombreuses, plus ou moins lexicalisées ; regroupement du vocabulaire en arbres représentant des relations générique/spécifique, partie/tout ou des réseaux ; variation lexicale d'ordres divers

- problèmes rencontrés : documentation rare, insatisfaisante ; difficulté à trouver des définitions; recoupement des notions imparfait; terminologie "floue»; profusion synonymique

\section{DESCRIPTION DU SÉMINAIRE DE TERMINOLOGIE}

\subsection{Le thème fixe}

Un seul thème est traité pendant la première moitié du trimestre ; ce sont les notions de base en lexicologie générale et technique, et, plus précisément, les questions de rédaction de définitions et d'organisation des documents lexicographiques. Le cours se compose d'une partie d'exposé, suivie d'exercices d'application. Il couvre la matière suivante :

1. Typologie des définitions

- définition par inclusion; structures générique/spécifique du lexique

- définition par faux-incluant; structures partie/tout du lexique

définition morpho-sémantique; structures morphologiques du lexique

- cas des définitions d'adjectifs et de verbes. 
2. Structures des ouvrages lexicographiques

Structures alphabétiques et systématiques. Regroupements morphologiques.

3. La phrase définitoire

Caractéristiques. Problème de traduction.

4. Les lexiques spécialisés

Définitions techniques typiques; adaptation de la définition suivant la clientèle visée ; défauts caractéristiques des lexiques techniques rédigés sans règle lexicographique stricte.

Les exercices consistent en des analyses de définitions choisies ponctuellement ou appartenant à des champs lexicaux limités et des comparaisons de définitions ; s'ajoutent quelques exercices de rédaction et de traduction de définitions. Enfin, on examine un certain nombre de lexiques techniques au niveau de leur structure et de la qualité de la rédaction des définitions.

\subsection{Les thèmes variables}

Suivant la composition du groupe d'étudiants, extrêmement variable d'une année sur l'autre, les thèmes suivants sont traités, cycliquement et plus ou moins longuement : 1. Les banques de termes : historique, fonctionnement ; exercices d'utilisation des banques accessibles aux étudiants.

2. La recherche terminologique ponctuelle : problèmes types, stratégies de recherche. Exercices sur des cas problèmes et rédaction de dossiers étoffés.

3. La normalisation : les organismes de normalisation, leur fonction, leur production ; historique des politiques linguistiques au Québec et au Canada ; bref aperçu du niveau international.

4. Étude comparative de quelques dictionnaires du français contemporain.

5. Morphologie et néologie : procédés de formation de termes en français; renouvellement des vocabulaires; stratégies d'implantation d'une nouvelle terminologie en contexte de traduction ou de francisation.

6. Étude approfondie de lexiques techniques divers.

\subsection{Les travaux}

La série de cours sur la définition est suivie d'un examen ou d'un travail pratique constitué de questions de synthèse et d'exercices d'application similaires à ceux faits en classe. Pendant la deuxième partie du trimestre, on demande aux étudiants de faire un travail de recherche sur corpus ou un travail de synthèse bibliographique, en rapport avec les thèmes traités au cours. Les étudiants qui s'orientent vers une recherche terminologique pour leur mémoire de maitrise peuvent présenter cette recherche comme travail de fin de trimestre. On leur demande un rapport sur la délimitation du domaine à couvrir, les problèmes posés par la terminologie à l'étude, le repérage documentaire, la nomenclature de départ et, enfin, sur la méthode générale de recherche. Les travaux de ces étudiants sont suivis en tutorat, en dehors des heures de cours.

En guise de conclusion, j'exprimerai un souhait qui, je l'espère, saura être exaucé : que les professeurs de terminologie continuent de décrire périodiquement le fonctionnement de leurs cours. La terminologie reste une discipline jeune, encore en cours d'élaboration, difficile à enseigner principalement à cause du manque de matériel pédagogique. Enseignée dans le cadre d'un programme de traduction, elle pose un double défi : concilier théorie et pratique, mais aussi donner une double formation aux étudiants, soit celle d'un terminologue producteur de travaux terminographiques et celle d'un traducteur principalement consommateur de ce genre de documents. Le domaine de la terminolo- 
gie n'a pas une très longue tradition académique ; les modèles pédagogiques demeurent donc extrêmement variables. Pour ma part, je viens d'exposer ici celui que j'ai utilisé en 1985-1986 ; il n'est pas parfait et il continuera d'évoluer au cours des prochaines années. J'espère simplement que mon texte saura alimenter la réflexion pédagogique.

\section{RÉFÉRENCES}

AUGER, Pierre (1979) : "L'enseignement de la terminologie (aspects théoriques et pratiques) dans le cadre des études en traduction et en linguistique ", Actes du $\sigma^{e}$ colloque international de terminologie. Pointe-auPic (Québec), 2-6 octobre 1977, Québec, OLF, pp. 445-484.

DUBUC, Robert (1975) : "Formation des terminologues - thériciens ou praticiens ", la Banque des mots, no 9, Paris, PUF, pp. 13-22.

DUBUC, Robert (1985) : "Cours type d'initiation à la terminologie ", TermNet News, 9, pp. 83-88.

PICHT, Heribert (1983) : "Training Terminologists for Term Banks ", Term Banks for Tomorrow's World. Translating and the Computer 4, London, Aslib, pp. 51-57.

ROBERTS, Roda (1985) : "The Teaching of Terminology and Documentation in Canadian Universities", TermNet News, 9, pp. 77-83.

SAGER, Juan Carlos (1981) : " La formation en terminologie : besoins, réalisations et perspectives d'avenir dans le monde ", Actes du colloque international sur l'enseignement de la terminologie. Université Laval, 28 au 30 août 1978, Québec, OLF, pp. 55-81.

SAGER, Juan Carlos (1981 et 1982) : "Terminologie et formation en terminologie ", Terminogramme, no 11, janvier 1981, pp. 1-4 et no 12 , mars 1982 , pp. 3-6. 